\title{
Radical prostatectomy versus brachytherapy for clinically localized prostate cancer on oncological and functional outcomes: a meta-analysis
}

\author{
Pu Zhang ${ }^{1 \#}$, Bei Qian ${ }^{2 \#}, J^{\prime i a w e i ~ S h i ~}{ }^{1 \#}$, Yajun Xiao ${ }^{1}$ \\ ${ }^{1}$ Department of Urology Surgery, ${ }^{2}$ Department of Plastic Surgery, Union Hospital, Tongji Medical College, Huazhong University of Science and \\ Technology, Wuhan 430022, China \\ Contributions: (I) Conception and design: YJ Xiao; (II) Administrative support: None; (III) Provision of study materials or patients: YJ Xiao; (IV) \\ Collection and assembly of data: P Zhang, B Qian, J Shi; (V) Data analysis and interpretation: B Qian, J Shi; (VI) Manuscript writing: All authors; (VII) \\ Final approval of manuscript: All authors. \\ \#These authors contributed equally to this work. \\ Correspondence to: Yajun Xiao. Department of Urology Surgery, Union Hospital, Tongji Medical College, Huazhong University of Science and \\ Technology, 1277 Jiefang Avenue, Wuhan 430022, China. Email: 15607123366@163.com.
}

\begin{abstract}
Background: Prostate cancer (PCa) is a form of malignancy that harms the health status of elderly men worldwide. It is unclear which of radical prostatectomy (RP) or brachytherapy (BT) is the more effective treatment for PCa. This study presents the first highly comprehensive and up-to-date comparative analysis of the overall outcomes of RP versus BT.

Methods: We conducted a systematic literature search for studies published on PubMed, EMBASE, and the Cochrane Library on the outcomes of RP versus BT in clinically localized PCa. The cumulative analysis was performed using Review Manager Version 5.3 software, and the Chi-square test was employed to test the statistical heterogeneity. The summary odds ratio (OR) and standard mean difference (SMD) was estimated using random effects models at $95 \%$ confidence intervals (CIs).

Results: In total, 2 randomized, 2 prospective, and 21 retrospective comparative studies were included. No significant differences in biochemical recurrence rate (BCR) (OR: 1.24; 95\% CI: 0.91, 1.68) and prostate cancer-specific mortality (PCSM) (OR: 1.62; 95\% CI: 0.86, 3.04) between RP and BT were noted. With erectile dysfunction and urinary incontinence, BT was more protective than RP in both short-term postoperative reports (OR: 2.06; 95\% CI: 1.15, 3.70 and OR: 4.62; 95\% CI: 2.33, 9.16) and long-term patient outcome reports (SMD: -5.62; 95\% CI: -13.81, 2.57 and SMD: -11.52 ; 95\% CI: $-18.32,-4.72$ ).

Conclusions: BT and RP for PCa therapy pose comparable risks of PCSM and BCR, while BT is associated with a lower incidence of erectile dysfunction and urinary incontinence. This study tentatively confirms that BT is an alternative to RP for patients seeking a curative treatment with minimal risks of urinary incontinence and sexual dysfunction.
\end{abstract}

Keywords: Prostate cancer (PCa); radical prostatectomy (RP); brachytherapy (BT); meta-analysis

Submitted Oct 25, 2019. Accepted for publication Jan 17, 2020.

doi: $10.21037 /$ tau.2020.02.15

View this article at: http://dx.doi.org/10.21037/tau.2020.02.15

\section{Introduction}

Prostate cancer (PCa) accounts for the highest number of newly diagnosed cancer cases among elderly men in developed countries (1). China and other developing countries are also recording annual increases in $\mathrm{PCa}$ incidence because of an upsurge in the aging population and changes in dietary patterns. PCa, therefore, represents a significant public global health concern (2). Unlike other 
forms of cancer, patients with localized PCa (characterized by the absence of an identifiable regional lymph node or distant metastasis) generally have a longer survival period (3). Consequently, the choice of an appropriate therapeutic strategy to reduce the risks of mortality and recurrence while improving the quality of life for PCa patients is of critical importance. According to the American National Academy of Medicine, the comparative efficiency of contemporary treatment options for localized PCa has been a top research priority (4). Currently, most of the patients diagnosed with prostate-specific antigen (PSA)detected $\mathrm{PCa}$ undergo conventional radical treatment encompassing either RP or radiotherapy (5). Prostatectomy (RP) is the standard of surgical treatment of localized $\mathrm{PCa}$ and entails the removal of the prostate and seminal vesicles, with or without pelvic lymphadenectomy $(6,7)$. Radiotherapy involves a combination of external-beam radiation therapy (EBRT), androgen deprivation therapy (ADT), and brachytherapy (BT) (8). Nevertheless, BT with implantation of radioisotopes such as iodine- 125 or palladium-103 is regarded as the recommended alternative for PCa treatment. BT is given as a single dose and is costeffective, making it a safe and feasible treatment option for outpatients (9). However, surveillance or active monitoring with deferred radical treatment is the most conservative option and can circumvent the need for an immediate and potentially unnecessary intervention (10). The lack of substantial information on the safety of this option, however, has limited its application. The Scandinavian Prostate Cancer Group 4 trial (SPCG-4) showed that RP decreased the risk of metastases and mortality rates from both PCa and any other cause (11). After an additional 3 -year follow-up, the SPCG-4 was more confident that RP reduced the mortality rate of death PCa patients (12). However, the first randomized comparison of active monitoring and radical treatment by the Prostate Testing for Cancer and Treatment (ProtecT) reported that surgery was not effective for patients with PSA-detected cancer (1). Although radical treatment gives patients a survival advantage, it is associated with side-effects such as urinary incontinence and erectile dysfunction, which may have a long-term impact on the quality of life $(4,13)$. To our knowledge, there is currently no sufficient evidence demonstrating the superiority of either RP or BT on both oncologic results and patient-reported outcomes (PRO). This study is thus the first highly comprehensive and up-todate meta-analysis and systematic review on this subject.

\section{Methods}

\section{Literature search}

A systematic literature search was performed using reports published in PubMed, EMBASE, and Cochrane Library according to the Preferred Reporting Items for Systematic Reviews and Meta-Analyses checklist (PRISMA). The search strategy involved a combination of the following medical subject headings (Mesh) keywords and free words: ((( prostate cancer) OR neoplasia) OR malignancy)) AND $(((()(()(($ Prostatectomies) OR Prostatectomies, Suprapubic) OR Suprapubic Prostatectomies) OR Suprapubic Prostatectomy) OR Prostatectomy, Retropubic) OR Prostatectomies, Retropubic) OR Retropubic Prostatectomies) OR Retropubic Prostatectomy) OR "Prostatectomy" [Mesh])) AND Brachytherapy) OR Curietherapy) OR Brachytherapy, Radioisotope) OR Plaque Therapy, Radioisotope) OR Radioisotope Plaque Therapy) OR Therapy, Radioisotope Plaque) OR Surface Radiotherapy) OR Radiotherapy, Surface) OR Radiotherapy, Intracavity) OR Intracavity Radiotherapy) OR Radiotherapy, Interstitial) OR Interstitial Radiotherapy) OR Radiotherapy, Implant) OR Implant Radiotherapy)) OR "Brachytherapy" [Mesh])). We also hand-searched the articles included in our reference list.

\section{Selection and exclusion criteria}

This study comprises publications that met the following criteria: (I) prospective or retrospective studies including full text or meeting abstract; (II) patients with localized PCa in the studies initially with RP or BT as major treatments; (III) studies providing data about the PRO and oncological outcomes of patients after BT or RP; (IV) articles published in English and involving human participants. The exclusion criteria were as follows: (I) reviews, abstracts, or letters; (II) studies with ambiguous results; (III) articles that could not be accessed. If either the timing or sources of the study population overlapped in 2 or more publications by the same authors, our review only included the most recent study or the study with the highest number of participants.

\section{Quality assessment}

Two reviewers (P Zhang and B Qian) independently assessed the quality of the study and the risk of bias of the included studies. The criteria detailed in the Cochrane 
Handbook 5.1.0 was used to evaluate randomized controlled trials (RCT), reporting of blinding, allocation concealment, withdrawals, and loss to follow-up. The nine-star Newcastle-Ottawa scale (NOS) quality assessment scale was used to evaluate the quality of comparative observational studies, for which a higher score indicated a better study quality (14). The quality of the research was estimated from the selection of study groups, comparability of groups, and assessment of outcomes; studies of the highest quality were awarded up to 9 stars. Any disagreements were resolved by consensus.

\section{Data extraction}

Data were independently retrieved by 2 investigators, P Zhang, B Qian. Any discrepancies between the 2 investigators' tallies were settled by incorporating a third investigator, JW Shi. For each study included, we extracted information including the name of the first author, year of publication, study design, country, the number of study participants, the clinical stage of patients, the value of PSA, the number of $P C a$-specific mortalities (PCSMs), biochemical recurrence, urinary incontinence, sexual dysfunction, and PRO of urinary incontinence posttreatment. Biochemical recurrence (PSA $>0.2 \mathrm{ng} / \mathrm{mL}$ for $\mathrm{PR}$ or $\mathrm{PSA}>$ nadir $+2 \mathrm{ng} / \mathrm{mL}$ for $\mathrm{BT}$ ) marked the primary endpoint of the study. The secondary endpoint was either urinary incontinence characterized by the need for one or more pads a day or sexual dysfunction defined by patients with postoperative International Index of Erectile Function (IIEF) $-5<17$.

\section{Statistical analysis}

The odds ratio $(\mathrm{OR})$ reflected the association between the choice of treatment and the risk of biochemical recurrence. The OR also indicated the association between the treatments and the risk of either urinary incontinence or sexual dysfunction. Review Manager Version 5.3 software (Cochrane Collaboration, Oxford, UK) designed for composing Cochrane Reviews, was employed for metaanalysis while the Chi-square test was used to assess statistical heterogeneity. The frequencies of biochemical recurrence, urinary incontinence, and erectile dysfunction following either prostatectomy or BT were determined using a fixed-effect pairwise meta-analysis. Random effects analysis was performed in case a significant statistical heterogeneity was observed. The $\mathrm{I}^{2}$ statistic was used as an indicator of percentage heterogeneity among studies and was interpreted as follows: $\mathrm{I}^{2}<50 \%$, low heterogeneity; $50-$ $75 \%$, moderate heterogeneity; $>75 \%$, high heterogeneity. The funnel plot asymmetry was used to assess the publication bias of a meta-analysis that included more than 10 studies.

\section{Results}

\section{Literature search}

Following a screening of the available databases, 1,756 potentially relevant publications were identified, including 1,486 from PubMed, 121 from EMBASE, and 149 from Cochrane Library. In total, 18 articles identified as duplicate search results were omitted, 1,702 studies were excluded by scanning titles and abstracts, and full texts of 36 citations were obtained. All the remaining articles, including their respective references, were carefully read according to the predefined inclusion and exclusion criteria. Ultimately, 25 studies were selected for the present meta-analysis. A flow diagram detailing the literature selection process is shown in Figure 1.

\section{Clinical characteristics of included studies}

A total of 2 RCT and 22 comparative observational studies were included in this analysis. In the studies, a total of 61,752 patients underwent primary radical treatment, with 45,002 being treated by RP and 16,750 by BT. Further characterization of the included studies is detailed in Table 1 $(15-29)$. All the studies were published within the last 2 decades. In terms of country of publication, 12 studies were conducted in the USA, while the remaining 13 studies were conducted in China, Spain, Italy, Canada, France, Germany, and The Netherlands. The number of participants in each treatment cohort varied from 93 to 41,395 . The maximum follow-up period for most of the groups in the meta-analysis was 13 years; 6 studies followed up patients for 5 years, and 3 studies followed up for 6 years. The other studies followed up patients ranging from one and a half years to 12 years. Most of the included studies were retrospective with a low evidence level. As a result, additional statistical analysis based on these data was performed.

\section{Quality of included studies}

Cochrane collaborative tools were used to assess the risk of 


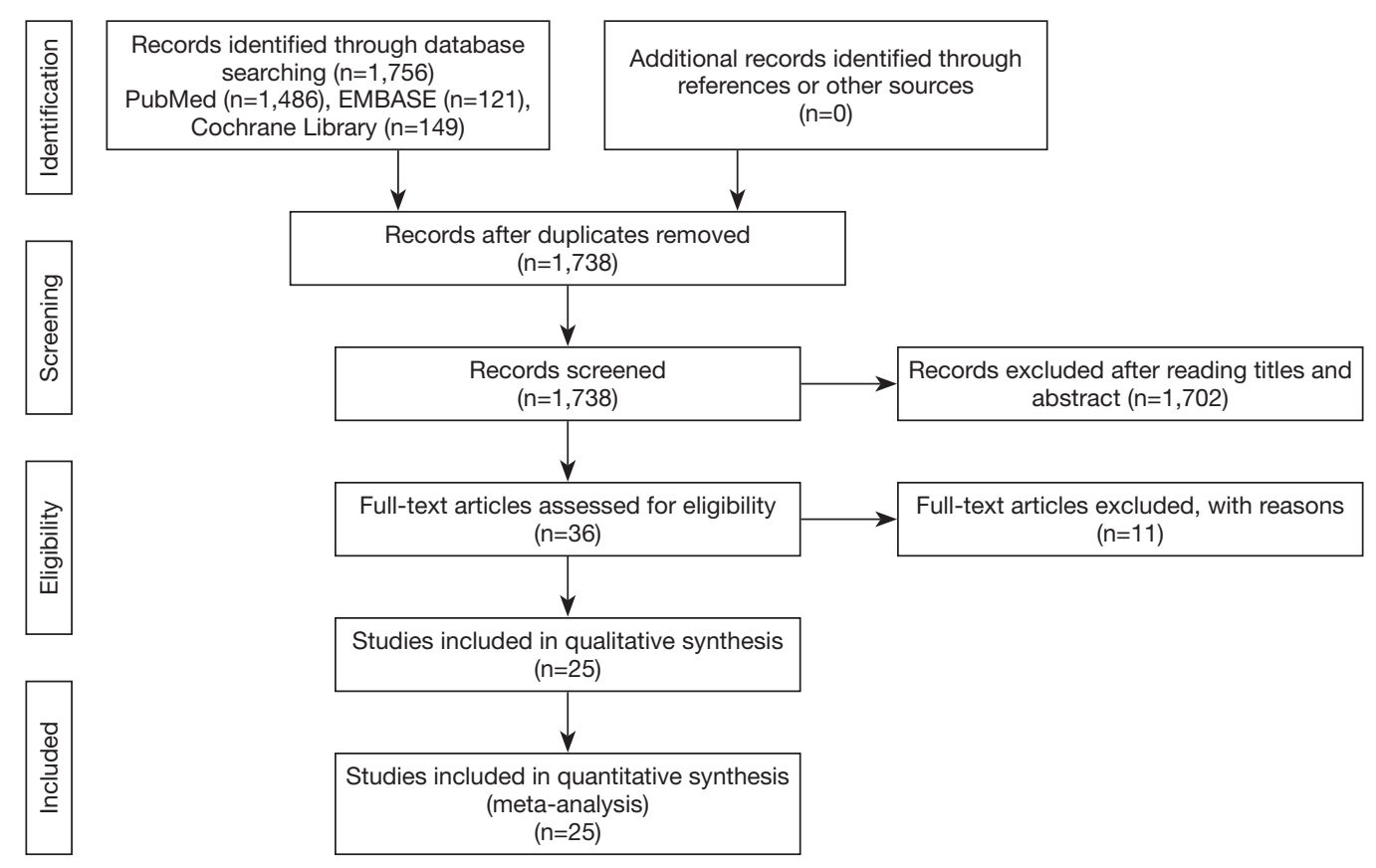

Figure 1 The flow chart of the selection process.

bias in the RCTs, and the results are presented in Figures 2,3. The NOS score adapted for prospective and retrospective cohort studies ranged from 5 to 7 . The results are displayed in Table 1. The overall quality of all the studies was medium, and the highest score was 9 .

\section{Prostate cancer-specific mortality (PCSM)}

Out of the 25 studies included, only Arvold et al., Potters et al., and Tward et al. reported PCSM while the remaining 22 studies compared BCR, urinary incontinence rate, and erectile dysfunction rate between RP and BT groups. A total of 51,713 patients were included in the studies. The results of this study showed that the PCSM rates of the 2 groups were $1.1 \%$ for $\mathrm{RP}$ and $0.5 \%$ for $\mathrm{BT}(\mathrm{OR}=1.62$; 95\% CI: 0.86-3.04), and there was no significant difference between the 2 groups $\left(\mathrm{P}=0.13, \mathrm{I}^{2}=59 \%\right)$ (Figure 4). The funnel plot showed that there were no significant deviations in PCSM rates.

\section{Biochemical recurrence rate (BCR)}

Estimation of the BCR of the 2 groups incorporated a total of 8,385 patients. The results showed that the BCR of the 2 groups was $16.8 \%$ and $15.9 \%$, respectively (OR $=1.24,95 \%$ CI: $0.91-1.68, \mathrm{P}=0.17)$. There was no statistically significant difference between the BCR of the 2 groups $(\mathrm{P}=0.17$, $\mathrm{I}^{2}=77 \%$, Figure 5). To further explore the heterogeneity among studies investigating the patients with BCR after these 2 treatments, we performed meta-regression analysis across various factors, including the sample size and followup years of studies, and the time of the studies conducted, and we found these factors were not significantly associated with the heterogeneity (Figure 6). The funnel plot in Figure 7 shows no significant deviation in BCR between the 2 groups.

\section{Urinary incontinence rate}

A total of 6 studies reported the urinary incontinence rate, and a total of 1,468 patients were enrolled in the reviews. The urinary incontinence rates in the RP and BT groups were $21.8 \%$ and $9.7 \%$, respectively $(\mathrm{OR}=4.62$; 95\% CI: 2.33-9.16). Significant differences in the urinary incontinence rate of the 2 groups were recorded $(\mathrm{P}<0.0001$, $\mathrm{I}^{2}=48 \%$, Figure 8 ).

\section{Erectile dysfunction rate}

The erectile dysfunction rate was obtained from 6 studies comprising a total of 1,001 patients. The meta-analysis showed that the erectile dysfunction rate in the RP and BT 


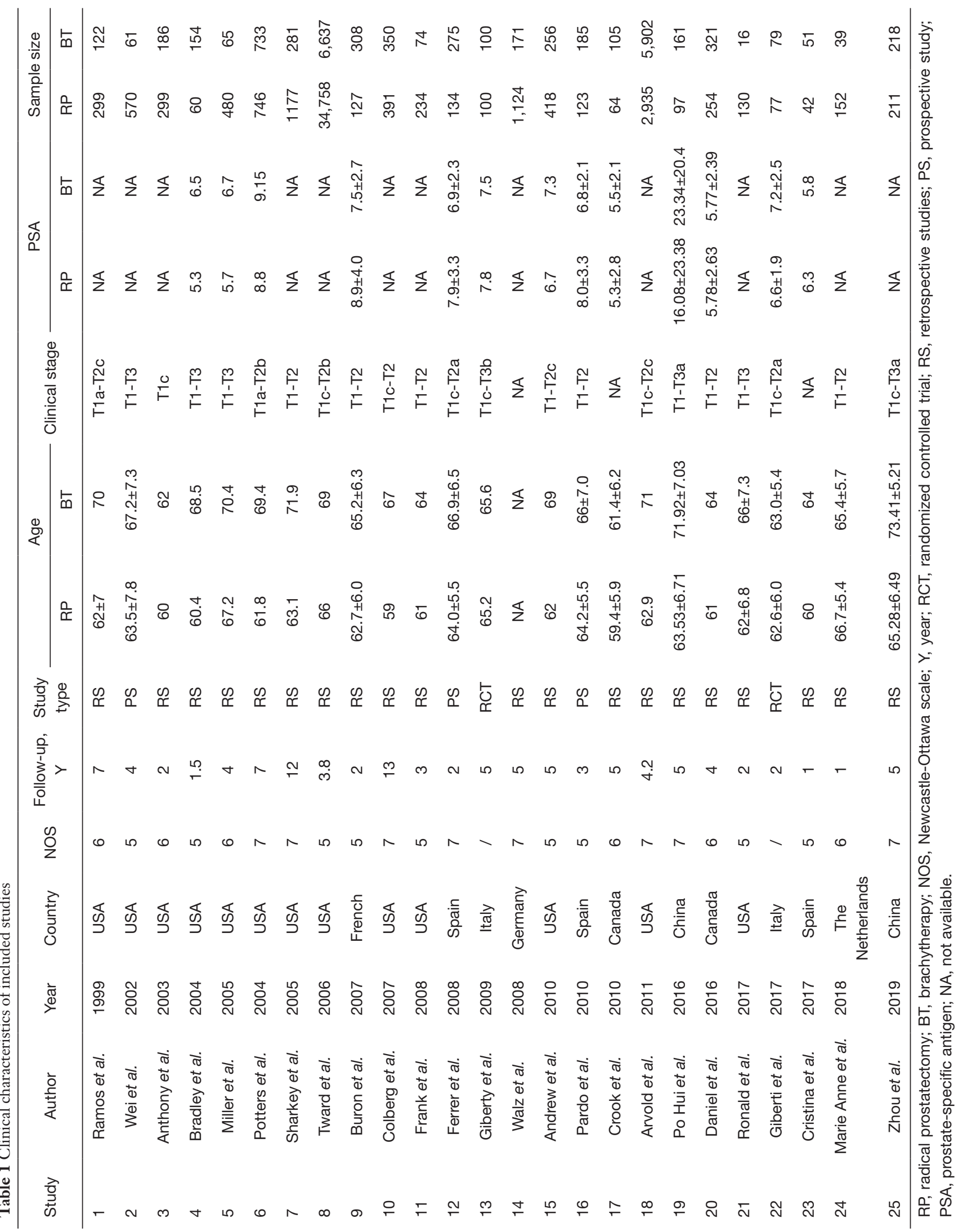




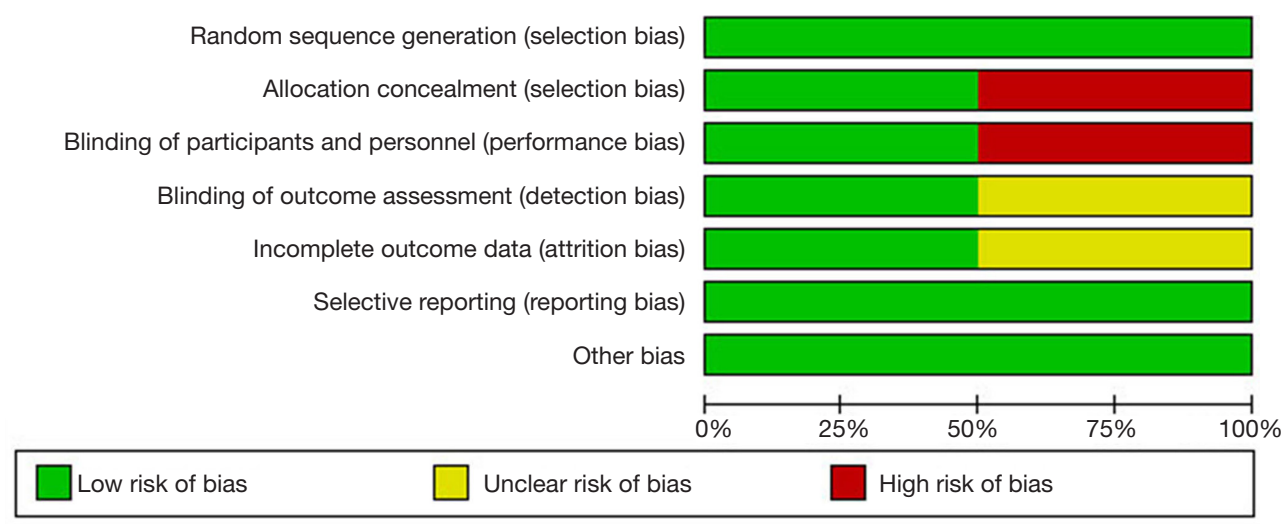

Figure 2 Risk of bias graph.

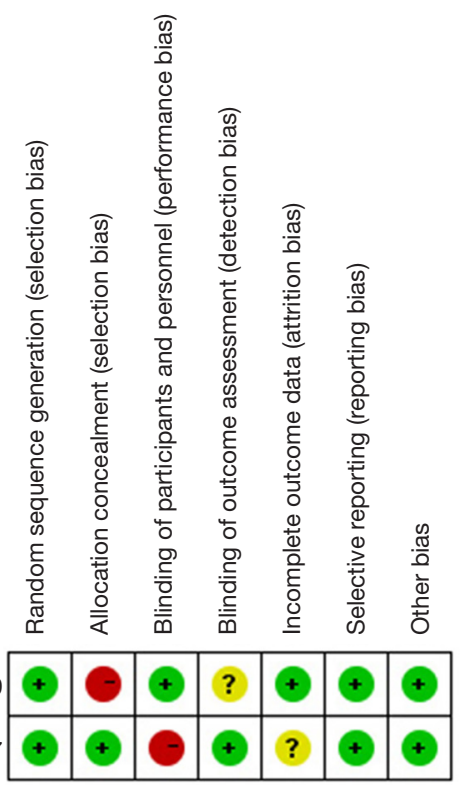

Figure 3 Risk of bias summary.

groups was $54.5 \%$ and $31.5 \%$, respectively (OR $=2.06 ; 95 \%$ CI: $1.15-3.70)$, and the difference between the 2 groups was statistically significant $\left(\mathrm{P}=0.002, \mathrm{I}^{2}=74 \%\right.$, Figure 9).

\section{PRO of urinary incontinence}

The PRO of urinary incontinence using Expanded Prostate Cancer Index Composite (EPIC) scores between RP and BT had a standard mean difference of (SMD) of -11.52 (95\% CI: -18.32-4.72). Significant differences in the EPIC scores of the 2 groups were recorded $\left(\mathrm{P}=0.0009, \mathrm{I}^{2}=87 \%\right.$, Figure 10).

\section{PRO of sexual function}

A total of 6 studies on PRO of sexual function were included in this meta-analysis. The results of this analysis showed that sexual function scores between the RP and BT groups had an SMD of -5.62 and a 95\% CI of $-13.82-2.57$. There was no significant difference in sexual functionality between the 2 groups ( $\mathrm{P}=0.18, \mathrm{I}^{2}=89 \%$ ) (Figure 10).

\section{Publication biases}

The BCR funnel plot for RP and BT is presented in Figure 11. A general overview of the image indicated that the distribution of each study in the triangle was relatively symmetrical. To be certain, we also used the Begg's test to evaluate and verify the statistical symmetry of the funnel plot by calculating the $\mathrm{P}$ value. The results are shown in Figure 12. $\mathrm{P}<0.05$ is regarded as a significant publication bias. The result reveals that the funnel plot was statistically symmetrical. There was, therefore, no significant publication bias in the study.

\section{Discussion}

PCa is the second most prevalent malignant tumor in men worldwide (30,31). Prostatectomy (RP), the current standard treatment for PCa patients, is highly effective in tumor control. However, RP has been associated with several adverse effects, such as the possibility of impaired sexual and urinary function, which decreases the quality of life for PCa patients (32). Consequently, several alternatives to PR, such as active surveillance, EBRT plus ADT, and BT, have been proposed. However, whether active 


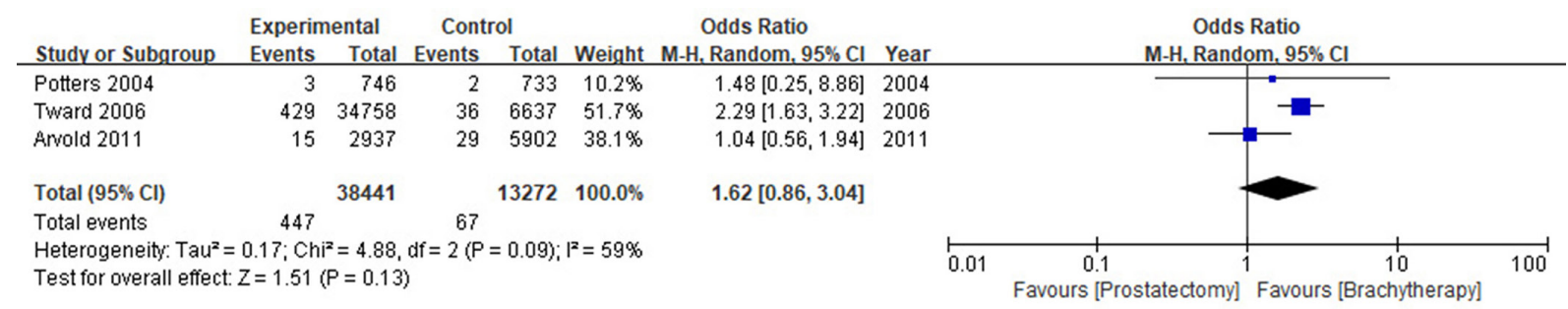

Figure 4 Forest plot of the PCSM rate between RP and BT. PCSM, prostate cancer-specific mortality; RP, radical prostatectomy; BT, brachytherapy.

\begin{tabular}{|c|c|c|c|c|c|c|c|c|c|c|c|c|}
\hline Study or Subgroup & \multicolumn{2}{|c|}{ Experimental } & \multicolumn{2}{|c|}{ Control } & \multicolumn{3}{|c|}{ Odds Ratio } & \multicolumn{5}{|c|}{$\begin{array}{c}\text { Odds Ratio } \\
\text { M-H, Random, } 95 \% \mathrm{Cl}\end{array}$} \\
\hline Ramos 1999 & 35 & 299 & 23 & 122 & $8.1 \%$ & $0.57[0.32,1.01]$ & 1999 & & & & & \\
\hline Anthony 2003 & 23 & 299 & 10 & 186 & $6.6 \%$ & $1.47[0.68,3.16]$ & 2003 & & & & & \\
\hline Bradley 2004 & 13 & 60 & 19 & 154 & $6.5 \%$ & $1.97[0.90,4.29]$ & 2004 & & & & & \\
\hline Potters 2004 & 157 & 746 & 190 & 733 & $10.6 \%$ & $0.76[0.60,0.97]$ & 2004 & & $\rightarrow$ & & & \\
\hline Sharkey 2005 & 63 & 281 & 130 & 1177 & $10.0 \%$ & $2.33[1.67,3.25]$ & 2005 & & & $\rightarrow$ & & \\
\hline Colberg 2007 & 64 & 391 & 53 & 350 & $9.6 \%$ & $1.10[0.74,1.63]$ & 2007 & & & & & \\
\hline Giberty 2009 & 8 & 100 & 7 & 100 & $4.8 \%$ & $1.16[0.40,3.32]$ & 2009 & & & & & \\
\hline Andrew 2010 & 97 & 418 & 27 & 256 & $9.1 \%$ & $2.56[1.62,4.06]$ & 2010 & & & $\rightarrow$ & & \\
\hline Walz 2010 & 158 & 1124 & 14 & 171 & $8.1 \%$ & $1.83[1.04,3.25]$ & 2010 & & & & & \\
\hline Daniel 2016 & 54 & 254 & 66 & 321 & $9.5 \%$ & $1.04[0.70,1.56]$ & 2016 & & & & & \\
\hline Po hui 2016 & 47 & 97 & 88 & 161 & $8.7 \%$ & $0.78[0.47,1.29]$ & 2016 & & - & & & \\
\hline Giberti 2017 & 2 & 77 & 3 & 79 & $2.3 \%$ & $0.68[0.11,4.16]$ & 2017 & & & & & \\
\hline zhien Zhou 2019 & 12 & 211 & 11 & 218 & $6.1 \%$ & $1.13[0.49,2.63]$ & 2019 & & & & & \\
\hline Total $(95 \% \mathrm{Cl})$ & & 4357 & & 4028 & $100.0 \%$ & $1.24[0.91,1.68]$ & & & & & & \\
\hline Total events & 733 & & 641 & & & & & & & & & \\
\hline $\begin{array}{l}\text { Heterogeneity: Tau } \\
\text { Test for overall effec }\end{array}$ & $\begin{array}{l}0.21 ; \mathrm{Chi} \\
\mathrm{Z}=1.36(\end{array}$ & $\begin{array}{l}=53.26 \\
=0.17)\end{array}$ & $\mathrm{df}=12$ & $(P<0 . C$ & $00001) ; 1^{2}$ & $=77 \%$ & & 0.01 & $\begin{array}{ll}0.1 & 1 \\
\text { Favours [RP] }\end{array}$ & Favours [ & $\begin{array}{r}10 \\
{[\mathrm{BT}]}\end{array}$ & 100 \\
\hline
\end{tabular}

Figure 5 Forest plot of BCR rate between RP and BT. BCR, biochemical recurrence rate; BT, brachytherapy.

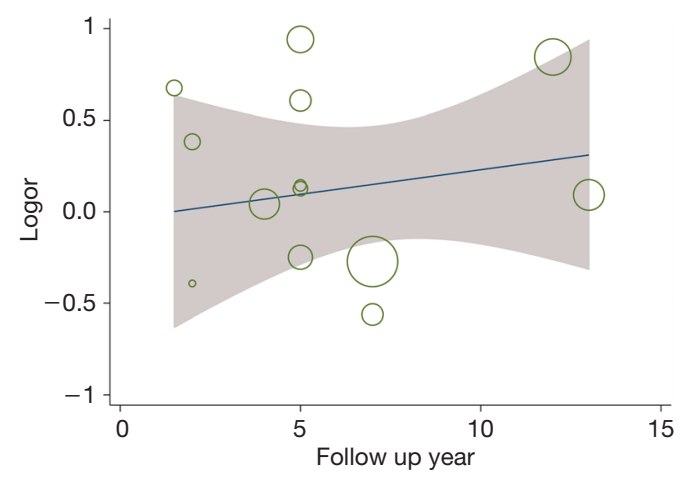

Figure 6 The meta-regression for follow-up years and BCR rate. $\mathrm{BCR}$, biochemical recurrence rate.

surveillance improves the survival and quality of life of patients remains to be explored $(1,12)$. The EBRT plus ADT treatment option has often been compared with RP, and various studies concluded that EBRT plus ADT could have the same tumor control effect as RP. This treatment

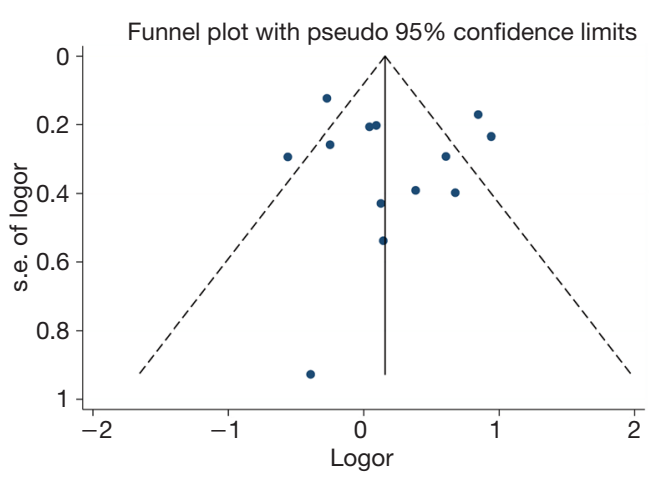

Figure 7 Funnel plot for primary outcome (BCR rate). BCR, biochemical recurrence rate.

option, however, demanded a long period of hospitalization and caused several complications including radiocystitis, urethrostenosis, intestinal dysfunction and irritability, and osteoporosis. Subsequently, some patients preferred the more convenient and economical BT which did not require 


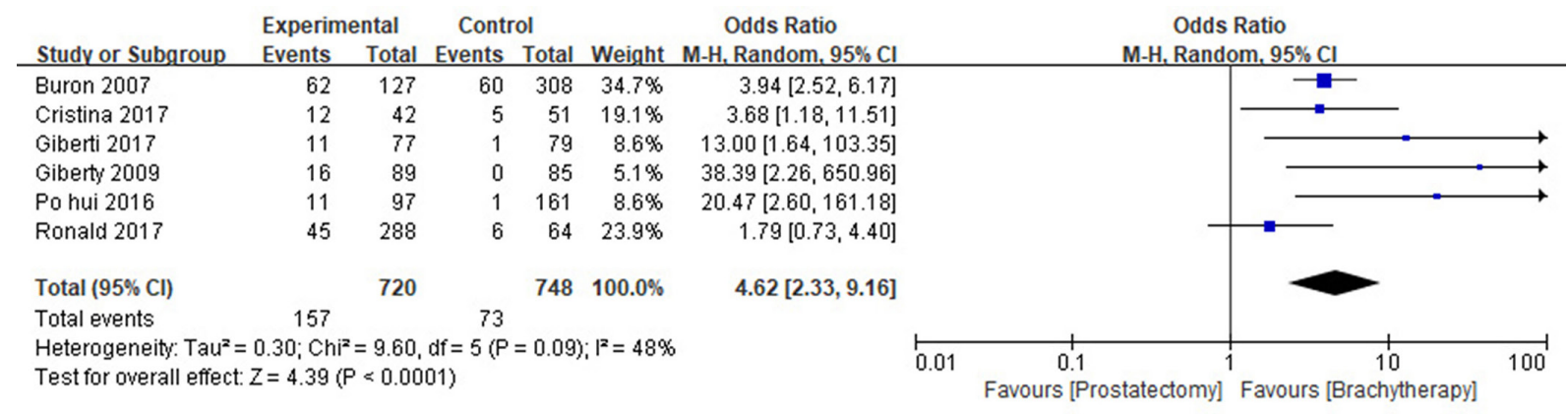

Figure 8 Forest plot of the urinary incontinence rate between RP and BT. RP, radical prostatectomy; BT, brachytherapy.

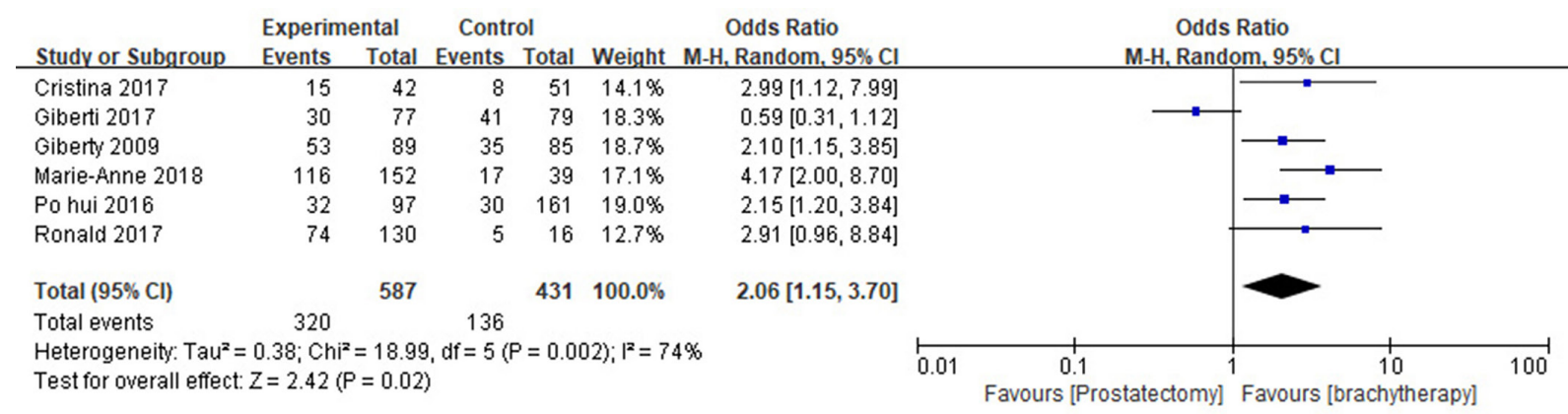

Figure 9 Forest plot of the erectile dysfunction rate between RP and BT. RP, radical prostatectomy; BT, brachytherapy.

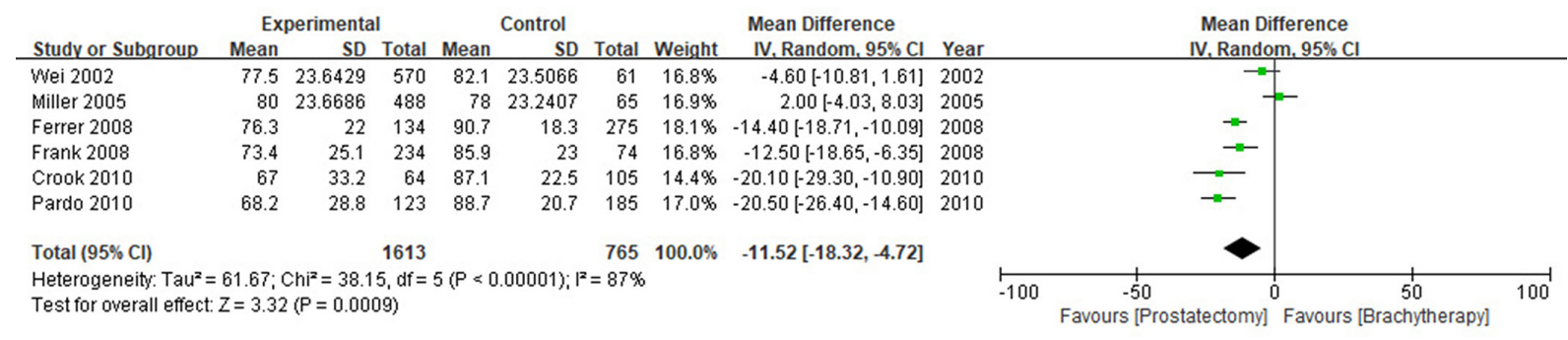

Figure 10 Forest plot of the PRO of urinary incontinence. PRO, patient-reported outcomes.

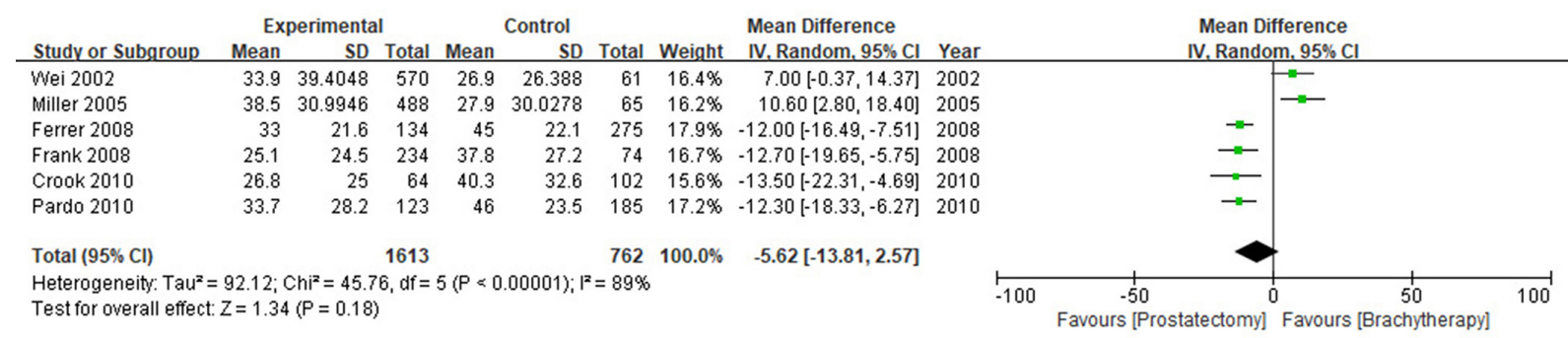

Figure 11 Forest plot of the PRO of sexual dysfunction. PRO, patient-reported outcomes. 


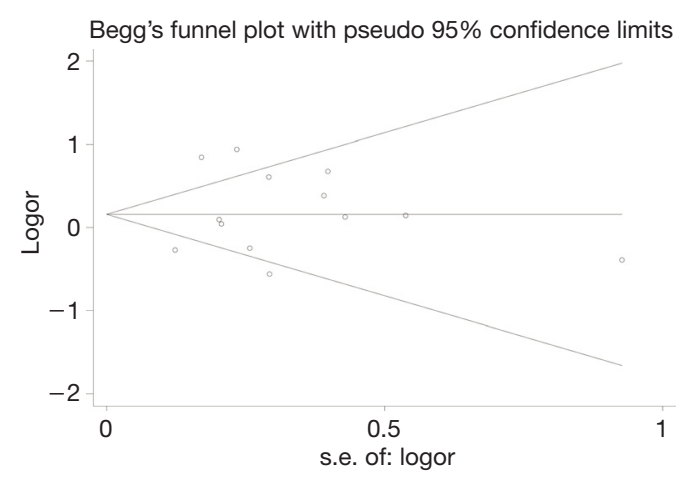

Figure 12 Begg's test for the studies investigating BCR rate. BCR, biochemical recurrence rate.

hospitalization. BT was reported to have an oncologic control capacity similar to that of RP, and better functional preservation than RP, while different studies reported the opposite outcome (33). From the perspectives of most doctors, the relative merits of BT and RP have not yet been made apparent. This uncertainty motivated the design of our review which compared the effectiveness of $\mathrm{BT}$ and $\mathrm{RP}$ on tumor control and functional preservation. This study forms the first highly comprehensive and up-to-date metaanalysis that simultaneously compares the oncological and functional outcomes of these 2 treatments.

This meta-analysis compared the BCR and PCSM rates of patients treated by $\mathrm{BT}$ versus those treated by RP. No significant differences in both the BCR (OR: 1.24; 95\% CI: 0.91, 1.68) and PCSM (OR: 1.62; 95\% CI: $0.86,3.04)$ between the 2 therapies were noted during the observation period. Previous studies have reported that BT selectively kills tumor cells with little effect on healthy tissues and was, therefore, a minimally invasive therapy for PCa. However, it has been reported that BT does not remove both the prostate gland and the surrounding potentially oncogenic tissues as thoroughly as RP does (34). Our findings indicated that BT was superior to RP on functional preservation. Our results revealed that patients treated via BT had much higher sexual dysfunction and urinary incontinence rates than those treated by BT in both short-term post-operative reports (OR: 2.06; $95 \%$ CI: $1.15,3.70$ and OR: 4.62 ; $95 \%$ CI: $2.33,9.16)$ and longterm PRO (OR: -5.62 ; 95\% CI: $-13.81,2.57$ and OR: -11.52 ; $95 \%$ CI: $-18.32,-4.72$ ), which was consistent with the expectations of many urologists and radiologists. RP involving the removal of the prostate and seminal vesicles is highly demanding for both surgeons and patients. Even surgeons who have performed thousands of prostatectomies may still damage the neurovascular bundle which is crucial to urinary and sexual function. One reason for the difficulty of this treatment is the anatomical complexity of the neurovascular bundle potentially being be attached to the prostate. In this condition, the prostate cannot be removed entirely without damaging the neurovascular bundles (35). Even after strictly adhering to the nervesparing RP suggested by Walsh, about $30 \%$ of patients suffered sexual dysfunction after the surgery. The failure indicated that the surgeon's knowledge about the anatomy of the neurovascular bundles was still insufficient, and the distribution of these bundles may not be limited to the side and back of the prostate, as described by Walsh (36). The neurovascular bundles could also be located at the front part of the prostate (37). For patients, at clinical stages, T2c, T3, or greater, or Gleason score $>7$, preservation of the neurovascular bundle was not recommended in cases where the positive margin could be found in the pathological report (38). Also, for patients where the preoperative evaluation allowed for the preservation of the neurovascular bundles, the localization of a tumor invasion on the pelvis fascia meant that neurovascular bundles should equally be removed (39). For this reason, it was difficult to protect the crucial neurovascular bundles during RP, especially due to the anatomical variations among patients. Other factors thought to be related to urinary incontinence include posterior fascia reconstruction, preservation of the bladder neck during surgery, and regular exercise of pelvic floor muscles (40). These factors further increase the complexity of RP, with surgeons expected to do their best to of preserve both the urinary and sexual functions of patients. On the contrary, BT provides a rapid dose diffusion at a distance from the radioactive source. As a result, the tissues surrounding the tumor are only exposed to limited doses of radiation and are thus protected from the immense damage that characterizes RP (41). A combination of these factors may explain why patients treated by BT could obtain better functional preservation than those treated with RP. To enhance the reliability and comprehensiveness of our study, we also integrated the PRO on the urinary incontinence and sexual function using EPIC scores. No significant differences between the 2 treatment options were observed in sexual function scores, but significant differences were observed in urinary incontinence scores. This may be explained by the fact that even though BT is a relatively minimally invasive treatment and causes less injury to the normal tissues compared to RP, the patients inevitably 
suffer from impairment in physical, cognitive, and social functions owing to persistent discomfort and complications such as bowel irritation which exert a negative effect on sexual function (22).

We included studies from different researchers treating patients in variable conditions, and a vast discrepancy was, therefore, predictable. Variations in age, race, and clinical stages of patients, preoperative serum concentration of PSA, the Gleason scores, preoperative urinary and sexual function, the surgeons' expertise in RP, the forms of RP adopted by surgeons, and implantation quality of BT could all lead to diverse outcomes. It has been reported that the patients diagnosed with $\mathrm{PCa}$ at a younger age may have poorer tumor differentiation which may lead to an adverse prognosis (42). Another example is that the patients with clinical stage $\leq \mathrm{T} 2$ were reported to have better outcomes in the BCR rate than that of patients with clinical stage $\geq \mathrm{T} 3$ after RP (43). Moreover, after RP, the BCR rate for patients with Gleason scores of $4+3$ was believed to be higher than that of patients with the Gleason scores of $3+4$ (44). In addition, the forms of RP could be divided into intrafascial, interfascial, and extrafascial ones, sharing the same name but actually leading to distinct outcomes in tumor control and functional preservation (45). Compared to the laparoscope, the use of the da Vinci surgical robot for radical prostatectomy (RP) in some qualified hospitals may also generate variability, owing to sufficient exposure and a shorter learning curve (46). Another possible cause of this discrepancy could be the use of different statistical methods by the various researchers of our included studies. For instance, we observed different definitions of BCR. Some researchers reported that patients might experience BCR when PSA $>0.5 \mathrm{ng} / \mathrm{mL}$ following BT, while others thought that PSA should exceed the PSA nadir $+2.0 \mathrm{ng} / \mathrm{mL}$. The observed discrepancy may contribute to a statistical difference and, thus, influence the findings of our study.

The present study indicates that BT is superior to RP in the preservation of urinary continence and sexual function, while it is similar in tumor control capacity; this does not suggest that BT is a perfect replacement for surgery since this study did not explore other aspects like the bowel irritation involved in these 2 treatments. Another additional factor that needs to be examined is the effect on the intestinal tract, mostly reported by patients receiving BT. Moreover, the studies included in this review are from 1999 to 2019, which was a significant period. Within these 2 decades, significant advances in both techniques and instrumentation have been achieved. Currently practicing urologists have, for instance, witnessed the transition from open RP to robotic-assisted RP, which offers better preservation of both urinary and sexual function. The outcomes of our study should, therefore, be verified by conducting larger RCTs that incorporate more contemporary techniques.

\section{Limitations}

There are several limitations to this study. Firstly, most of the included studies were either case series or prospective cohort studies, and both case-control and randomization were absent in most of the included studies. Secondly, irritation of the intestinal tract, another crucial complication of BT, was not involved in our article, but this factor can greatly influence the quality of life of patients. Owing to the lack of comparative data in this field, the conclusions of this study may be limited. Further standardized studies involving large cohorts and randomized design should, therefore, be conducted to obtain more accurate results.

\section{Conclusions}

BT for PCa was associated with a similar risk of PCSM and BCR compared with RP, and a lower incidence of sexual dysfunction and urinary incontinence. This study tentatively confirms BT as an alternative to RP for patients seeking a curative treatment with minimal risks of urinary incontinence and sexual dysfunction. Additionally, more large samples and multi-center studies with standardized reports of perioperative parameters and clinical outcomes are needed for further evaluation in the future.

\section{Acknowledgments}

Funding: None.

\section{Footnote}

Conflicts of Interest: All authors have completed the ICMJE uniform disclosure form (available at http://dx.doi. org/10.21037/tau.2020.02.15). The authors have no conflicts of interest to declare.

Ethical Statement: The authors are accountable for all aspects of the work in ensuring that questions related to the accuracy or integrity of any part of the work are appropriately investigated and resolved. 
Open Access Statement: This is an Open Access article distributed in accordance with the Creative Commons Attribution-NonCommercial-NoDerivs 4.0 International License (CC BY-NC-ND 4.0), which permits the noncommercial replication and distribution of the article with the strict proviso that no changes or edits are made and the original work is properly cited (including links to both the formal publication through the relevant DOI and the license). See: https://creativecommons.org/licenses/by-ncnd/4.0/.

\section{References}

1. Donovan JL, Hamdy FC, Lane JA, et al. Patient-Reported Outcomes after Monitoring, Surgery, or Radiotherapy for Prostate Cancer. N Engl J Med 2016;375:1425-37.

2. Kimura T, Egawa S. Epidemiology of prostate cancer in Asian countries. Int J Urol 2018;25:524-31.

3. Hamdy FC, Donovan JL, Lane JA, et al. 10-Year Outcomes after Monitoring, Surgery, or Radiotherapy for Localized Prostate Cancer. N Engl J Med 2016;375:1415-24.

4. Chen RC, Basak R, Meyer AM, et al. Association Between Choice of Radical Prostatectomy, External Beam Radiotherapy, Brachytherapy, or Active Surveillance and Patient-Reported Quality of Life Among Men With Localized Prostate Cancer. JAMA 2017;317:1141-50.

5. Lane JA, Donovan JL, Davis M, et al. Active monitoring, radical prostatectomy, or radiotherapy for localised prostate cancer: study design and diagnostic and baseline results of the ProtecT randomised phase 3 trial. Lancet Oncol 2014;15:1109-18.

6. Chen RC, Chang P, Vetter RJ, et al. Recommended patient-reported core set of symptoms to measure in prostate cancer treatment trials. J Natl Cancer Inst 2014. doi: 10.1093/jnci/dju132.

7. Lowrance WT, Eastham JA, Savage C, et al. Contemporary open and robotic radical prostatectomy practice patterns among urologists in the United States. J Urol 2012;187:2087-92.

8. Whiting PF, Moore TH, Jameson CM, et al. Symptomatic and quality-of-life outcomes after treatment for clinically localised prostate cancer: a systematic review. BJU Int 2016;118:193-204.

9. Blasko JC, Ragde H, Luse RW, et al. Should brachytherapy be considered a therapeutic option in localized prostate cancer? Urol Clin North Am 1996;23:633-50.

10. Chen RC, Rumble RB, Loblaw DA, et al. Active
Surveillance for the Management of Localized Prostate Cancer (Cancer Care Ontario Guideline): American Society of Clinical Oncology Clinical Practice Guideline Endorsement. J Clin Oncol 2016;34:2182-90.

11. Bill-Axelson A, Holmberg L, Filen F, et al. Radical prostatectomy versus watchful waiting in localized prostate cancer: the Scandinavian prostate cancer group-4 randomized trial. J Natl Cancer Inst 2008;100:1144-54.

12. Bill-Axelson A, Holmberg L, Ruutu M, et al. Radical prostatectomy versus watchful waiting in early prostate cancer. N Engl J Med 2011;364:1708-17.

13. King MT, Viney R, Smith DP, et al. Survival gains needed to offset persistent adverse treatment effects in localised prostate cancer. Br J Cancer 2012;106:638-45.

14. Stang A. Critical evaluation of the Newcastle-Ottawa scale for the assessment of the quality of nonrandomized studies in meta-analyses. European Journal of Epidemiology 2010;25:603-5.

15. Zhou Z, Yan W, Zhou Y, et al. (125)I low-dose-rate prostate brachytherapy and radical prostatectomy in patients with prostate cancer. Oncol Lett 2019;18:72-80.

16. van Stam MA, Aaronson NK, Bosch J, et al. Patientreported Outcomes Following Treatment of Localised Prostate Cancer and Their Association with Regret About Treatment Choices. Eur Urol Oncol 2020;3:21-31.

17. Giberti C, Gallo F, Schenone M, et al. Robotic prostatectomy versus brachytherapy for the treatment of low risk prostate cancer. Can J Urol 2017;24:8728-33.

18. García-Sánchez C, Martin AA, Conde-Sanchez JM, et al. Comparative analysis of short - term functional outcomes and quality of life in a prospective series of brachytherapy and Da Vinci robotic prostatectomy. Int Braz J Urol 2017;43:216-23.

19. Chiang PH, Liu YY. Comparisons of oncological and functional outcomes among radical retropubic prostatectomy, high dose rate brachytherapy, cryoablation and high-intensity focused ultrasound for localized prostate cancer. Springerplus 2016;5:1905.

20. Taussky D, Ouellet V, Delouya G, et al. A comparative study of radical prostatectomy and permanent seed brachytherapy for low- and intermediate-risk prostate cancer. Can Urol Assoc J 2016;10:246-50.

21. Vassil AD, Murphy ES, Reddy CA, et al. Five year biochemical recurrence free survival for intermediate risk prostate cancer after radical prostatectomy, external beam radiation therapy or permanent seed implantation. Urology 2010;76:1251-7.

22. Giberti C, Chiono L, Gallo F, et al. Radical retropubic 
prostatectomy versus brachytherapy for low-risk prostatic cancer: a prospective study. World J Urol 2009;27:607-12.

23. Buron C, Le Vu B, Cosset JM, et al. Brachytherapy versus prostatectomy in localized prostate cancer: results of a French multicenter prospective medico-economic study. Int J Radiat Oncol Biol Phys 2007;67:812-22.

24. Bradley EB, Bissonette EA, Theodorescu D. Determinants of long-term quality of life and voiding function of patients treated with radical prostatectomy or permanent brachytherapy for prostate cancer. BJU Int 2004;94:1003-9.

25. Sharkey J, Cantor A, Solc Z, et al. 103Pd brachytherapy versus radical prostatectomy in patients with clinically localized prostate cancer: a 12-year experience from a single group practice. Brachytherapy 2005;4:34-44.

26. D'amico AV, Tempany CM, Schultz D, et al. Comparing PSA outcome after radical prostatectomy or magnetic resonance imaging-guided partial prostatic irradiation in select patients with clinically localized adenocarcinoma of the prostate. Urology 2003;62:1063-7.

27. Ramos CG, Carvalhal GF, Smith DS, et al. Retrospective comparison of radical retropubic prostatectomy and 125 iodine brachytherapy for localized prostate cancer. J Urol 1999;161:1212-5.

28. Potters L, Klein EA, Kattan MW, et al. Monotherapy for stage T1-T2 prostate cancer: radical prostatectomy, external beam radiotherapy, or permanent seed implantation. Radiother Oncol 2004;71:29-33.

29. Walz C, Joniau S, Salem N, et al. A matched-pair analysis between radical prostatectomy and low dose rate brachytherapy in low risk prostate cancer patients. Eur Urol 2008;7:167.

30. Galper SL, Chen MH, Catalona WJ, et al. Evidence to support a continued stage migration and decrease in prostate cancer specific mortality. J Urol 2006;175:907-12.

31. Mullins JK, Feng Z, Trock BJ, et al. The impact of anatomical radical retropubic prostatectomy on cancer control: the 30-year anniversary. J Urol 2012;188:2219-24.

32. Bolla M, Gonzalez D, Warde P, et al. Improved survival in patients with locally advanced prostate cancer treated with radiotherapy and goserelin. N Engl J Med 1997;337:295-300.

33. Ennis RD, Hu L, Ryemon SN, et al. Brachytherapy-Based Radiotherapy and Radical Prostatectomy Are Associated With Similar Survival in High-Risk Localized Prostate Cancer. J Clin Oncol 2018;36:1192-8.

34. Tanaka N, Asakawa I, Hasegawa M, et al. Low-dose-rate brachytherapy for prostate cancer: A 15 -year experience in
Japan. Int J Urol 2020;27:17-23.

35. Cocci A, Cito G, Romano A, et al. Radical prostatectomy and simultaneous penile prosthesis implantation: a narrative review. Int J Impot Res 2019. [Epub ahead of print].

36. Walsh PC. Anatomic radical prostatectomy: Evolution of the surgical technique. J Urol 1998;160:2418-24.

37. Eichelberg C, Erbersdobler A, Michl U, et al. Nerve distribution along the prostatic capsule. Eur Urol 2007;51:105-10; discussion 110-1.

38. Greco F, Hoda MR, Wagner S, et al. Bilateral vs unilateral laparoscopic intrafascial nerve-sparing radical prostatectomy: evaluation of surgical and functional outcomes in 457 patients. BJU Int 2011;108:583-7.

39. Kania P, Woskowiak P, Salagierski M. Preservation of continence in radical prostatectomy patients: a laparoscopic surgeon's perspective. Cent European J Urol 2019;72:32-8.

40. Lavigueur-Blouin H, Noriega AC, Valdivieso R, et al. Predictors of early continence following robot-assisted radical prostatectomy. Can Urol Assoc J 2015;9:e93-7.

41. Chargari C, Deutsch E, Blanchard P, et al. Brachytherapy: An overview for clinicians. CA Cancer J Clin 2019;69:386-401.

42. Johnson DE, Lanieri JP Jr, Ayala AG. Prostatic adenocarcinoma occurring in men under 50 years of age. J Surg Oncol 1972;4:207-16.

43. Theiss M, Wirth MP, Manseck A, et al. Prognostic significance of capsular invasion and capsular penetration in patients with clinically localized prostate cancer undergoing radical prostatectomy. Prostate 1995;27:13-7.

44. Sakr WA, Tefilli MV, Grignon DJ, et al. Gleason score 7 prostate cancer: a heterogeneous entity? Correlation with pathologic parameters and disease-free survival. Urology 2000;56:730-4.

45. Plas E, Riedl CR, Pfluger H. Malignant mesothelioma of the tunica vaginalis testis: review of the literature and assessment of prognostic parameters. Cancer 1998;83:2437-46.

46. Ahlering TE, Skarecky D, Lee D, et al. Successful transfer of open surgical skills to a laparoscopic environment using a robotic interface: initial experience with laparoscopic radical prostatectomy. J Urol 2003;170:1738-41.

Cite this article as: Zhang $\mathrm{P}$, Qian B, Shi J, Xiao Y. Radical prostatectomy versus brachytherapy for clinically localized prostate cancer on oncological and functional outcomes: a metaanalysis. Transl Androl Urol 2020;9(2):332-343. doi: 10.21037/ tau.2020.02.15 